\title{
Complex Dynamical Behaviors of a Mixed Duopoly Game Based on Intellectual Property Rights Protection
}

\author{
Tong Chu $\mathbb{D}^{1},{ }^{1}$ Yu Yu, ${ }^{2}$ and Xiaoxue Wang ${ }^{3}$ \\ ${ }^{1}$ School of Law, Zhejiang University of Finance and Economics, Hangzhou 310018, Zhejiang, China \\ ${ }^{2}$ School of Economics, Zhejiang University of Finance and Economics, Hangzhou 310018, Zhejiang, China \\ ${ }^{3}$ Research Centre of Game Theory and Economics Mathematics, Lanzhou Jiaotong University, Lanzhou 730070, Gansu, China \\ Correspondence should be addressed to Tong Chu; chutong@zufe.edu.cn
}

Received 26 June 2020; Revised 10 August 2020; Accepted 14 August 2020; Published 28 August 2020

Academic Editor: Tingqiang Chen

Copyright (C) 2020 Tong Chu et al. This is an open access article distributed under the Creative Commons Attribution License, which permits unrestricted use, distribution, and reproduction in any medium, provided the original work is properly cited.

Based on the oligopoly game theory and the intellectual property rights protection policy, we investigate the complex dynamical behaviors of a mixed duopoly game with quadratic cost. In the new system, a few parameters are improved by considering intellectual property rights protection and the stability conditions of the Nash equilibrium point are discussed in detail. A set of the two-dimensional bifurcation diagrams is demonstrated by using numerical modeling, and these diagrams show abundant complex dynamical behaviors, such as coexistence of attractors, different bifurcation, and fractal structures. These dynamical properties can present the long-run effects of strengthening intellectual property protection.

\section{Introduction}

Recently, a large number of research works about the duopoly game have been investigated. Among them, many scholars focus their attention on the complex dynamical behaviors of the duopoly game model [1-4], duopoly game with long-memory effects [5], R\&D (the abbreviation of research and development) competitions with spillover [6], and some new investigation in the R\&D evolution network model with spillover [7, 8]. Xin et al.[9-11] proposed a differential oligopoly game for optimal production planning and water savings, and analyzed the impact of oligopoly competition on the social welfare. Different optimal solutions with varying parameters are also simulated numerically. One main feature is that the developed countries will supply allowances to high-tech enterprises for R\&D activities. It is well recognized among scholars that the innovation in developed countries could generate externality, see Ref. [12] and Refs. [13, 14]. Liao and Wong [15] investigated a competition between the developed and the developing countries by considering two cases, where both R\&D allowance and intellectual property rights protection either at the same time or in turn were chosen to make an individual welfare or entire welfare maximization. That is to say, executing intellectual property rights protection in the developing countries could improve welfare of the world. Besides the ways that exchange trade policy and political intervention, the developed countries still have methods to prohibit protection on intellectual property rights from developing countries. In the empirical study of innovation and technological change, patent-based measurement is often used as an indicator. Especially, in the pharmaceutical industry, many empirical research studies showed that timing plays an important role in the R\&D activities, see Ref. [16], where the authors observe an influence coefficient named cost of "inventing around," this coefficient has a positive relation with speed of commodity launch, and it can be measured by the number of patent applications for a specific candidate drug. And the research of Cockburn et al. [17] indicated that both patent and price regulation will have a great impact on marketing of new drugs in a lot of countries or areas. There are numerous uncertain factors in intellectual property rights and market existing in the drug manufacturing industry. When enterprises face uncertainties in markets, they had no choice but to investigate policy of intellectual property rights so as to choose optimal 
R\&D timing. Billette de Villemeur et al. [18] built a model aiming at simulating dynamical competition among pharmaceutical enterprises under the frame of intellectual property rights. Their research results showed that enterprises can take advantages from open standards, and the effects of takeovers on social welfare (shorted as SW) are ambiguous. However, technical licensing is benefit to social welfare. In addition, when roles of enterprise are predetermined, the social welfare under protection of intellectual property is greater than that of endogenous innovation, which illustrates the significance of dynamic competition. Chu [19] indicates the significance of that pharmaceutical intellectual property rights protection in drug regulatory process. It could take positive effect on encouraging drug innovation and marketing so as to increasing social welfare in health service.

In general, dynamic competition among nationalized enterprises, nonnationalized enterprises, and foreign invested enterprises in the market could be regarded as a typical instance of oligopoly competition, which is a socalled mixed duopoly game model. Bárcena-Ruiz and Garzón [20] discussed the privatization of nationalized enterprises and found that the vertical integration of mixed duopoly is much lower. Gutiérrez-Hita and Vicente-Pérez [21] established a mixed duopoly model where the enterprises produce differentiated products and finally found that consumer surplus will increase with the products become more substitutive. In Ref. [22], from two aspects that market and administrative supervision mechanism and the view of evolving game, the scholar Yang discussed cooperating activities on intellectual property and corresponding effecting elements and pointed that it is critical for enterprises to execute intellectual property cooperation to integrate resources and increase level of innovation.

The remaining of this literature is listed as follows. A mixed duopoly game model is established in Section 2. Section 3 analyzes local stability properties of the equilibria by using nonlinear dynamical evolution theory and numerical simulation. Section 4 gives global dynamics analyzes, and some conclusions are given in Section 5.

\section{Establishment of Mixed Duopoly Game Model}

Considering two quantity-setting enterprises exists in a homogeneous product market, where the two enterprises are labeled by enterprise 1 (or N) and enterprise 2 (or S), respectively. Suppose the yields of enterprises are denoted as $q_{1}$ and $q_{2}$. And prices of the goods manufactured by these enterprises are separately labeled as $p_{1}$ and $p_{2}$, which is determined by following inverse demand function:

$$
p_{i}=a-b Q, \quad i=1,2,
$$

where $Q=q_{1}+q_{2}$ is total supply of this product to the market and $a>0$ and $b>0$ are determined by the elasticity of market supply and demand, respectively. Obviously, the prices of the products produced by these two enterprises are the same in the market. It means that $p_{1}=p_{2}=p$. And the cost functions of these two enterprises are given by

$$
C_{i}=c q_{i}^{2}, \quad i=1,2,
$$

where $c>0$ and $a>c$ are always satisfied. Through the above discussions, the profit function of enterprise $i$ can be easily obtained, which is given as

$$
\pi_{i}=\left[a-b\left(q_{1}+q_{2}\right)\right] q_{i}-c q_{i}^{2}, \quad i=1,2 .
$$

In the actual market, what the enterprise pursues is not only its own profit, but also its social responsibility. In this research, we take the social welfare (SW for short) as the reference index of corporate social responsibility. It is generally known that SW is sum of CS and profits of all enterprises. According to Cao et al. [4], the function of CS could be obtained as

$$
\mathrm{CS}=\int_{p}^{a} \frac{a-\bar{p}}{b} \mathrm{~d} \bar{p}=\frac{b\left(q_{1}+q_{2}\right)^{2}}{2},
$$

and $\bar{p} \in(p, a)$ is a variable with respect to price. Based on assumptions mentioned above, a specific form of social welfare can be gained by combining equations (3) and (4), which is given as

$$
\mathrm{SW}=a\left(q_{1}+q_{2}\right)-\frac{b}{2}\left(q_{1}+q_{2}\right)^{2}-c\left(q_{1}^{2}+q_{2}^{2}\right) .
$$

If we assume that both enterprises are semi-public ownership, then the enterprise will take its own revenue as well as the social welfare into its own objective. And thus, the objective of enterprise $i$ could be represented as

$$
\mathrm{O}_{i}=\left(1-\alpha_{i}\right) \pi_{i}+\alpha_{i} \mathrm{SW},
$$

where $0 \leq \alpha_{i} \leq 1, i=1,2$, is level of nationalization of enterprise $i$. Substituting equations (3) and (5) into equation (6), then the specific form of objective function of enterprise $i$ can be rewritten as

$$
\begin{aligned}
O_{i}= & \left(a-b\left(q_{1}+q_{2}\right)\right) q_{i}-c q_{i}^{2} \\
& +\alpha_{i}\left(\frac{b}{2}\left(q_{1}+q_{2}\right)^{2}+\left(a-b\left(q_{1}+q_{2}\right)\right) q_{j}-c q_{j}^{2}\right), \quad i \neq j,
\end{aligned}
$$

and we can calculate the first-order condition of objective function $O_{i}$ as

$$
\frac{\partial O_{i}}{\partial q_{i}}=a-b q_{j}-\left(2 b+2 c-\alpha_{i} b\right) q_{i}, \quad i=1,2, i \neq j .
$$

As the enterprises do not have complete information about its rivasl and the market, the enterprises in the market are often bounded rational. When the bounded rational enterprises make decisions at the same time, they can only determine their outputs in the next period through calculating the partial estimation of profit margin in the previous period. This particular dynamic decision mechanism is also called dynamic gradient adjustment mechanism by other 
scholars (see [23] for details). According to the so-called gradient adjustment mechanism, the dynamic decisionmaking process of these two enterprises can be described by the following difference equation:

$$
q_{i}(t+1)=q_{i}(t)+v_{i} q_{i} \frac{\partial O_{i}}{\partial q_{i}},
$$

where parameter $v_{i}>0$ is the enterprise $i$ s speed of adjustment. From the above dynamic adjustment process, we can see that enterprise $i$ will rise its own outputs in the next period when its marginal objective in the period $t$ is greater than zero. On the contrary, enterprise $i$ will reduce its own outputs in the next period when its marginal objective in period $t$ is less than zero. Taking equation (8) into equation (9), the specific expressions can be represented by

$$
T:\left\{\begin{array}{l}
q_{1}(t+1)=q_{1}(t)+v_{1} q_{1}(t)\left[a-b q_{2}(t)-\left(2 b+2 c-\alpha_{1} b\right) q_{1}(t)\right], \\
q_{2}(t+1)=q_{2}(t)+v_{2} q_{2}(t)\left[a-b q_{1}(t)-\left(2 b+2 c-\alpha_{2} b\right) q_{2}(t)\right],
\end{array}\right.
$$

or equivalent to

$$
T:\left\{\begin{array}{l}
q_{1}^{\prime}=q_{1}+v_{1} q_{1}\left[a-b q_{2}-\left(2 b+2 c-\alpha_{1} b\right) q_{1}\right] \\
q_{2}^{\prime}=q_{2}+v_{2} q_{2}\left[a-b q_{1}-\left(2 b+2 c-\alpha_{2} b\right) q_{2}\right]
\end{array}\right.
$$

where ${ }^{\prime}$ is the one-step time-advancement operator.

In equation (9), $v_{i}>0$ is the enterprise $i$ 's speed of adjustment, this parameter is affected by many factors, such as innovation potential, patent rights, copyrights, trademarks, and trade secrets. Barbu and Militaru [24] investigate the moderating effect of intellectual property rights on relationship between innovation and company performance in the manufacturing sector, and the findings indicate a positive correlation between patenting and new ventures' growth, access to venture capital, and new knowledge. The conceptual model was analyzed using hierarchical linear regression with company performance as the dependent variable and innovation potential, patent rights, copyrights, trademarks, and trade secrets as independent variables. And they also present a correlation matrix between latent variables with descriptive statistics in a questionnaire. In this paper, these research results can be used for reference of the value of the enterprise adjustment speed parameters owing to intellectual property rights protection.

Strengthening intellectual property rights protection does have definite effects on competition strategies of hightech enterprise. Furthermore, strengthening intellectual property rights protection also directly determines that enterprises whether transfer their production plant to a relative less-developed area. While in developed countries, R\&D activities will be influenced by inducement of imitations, which changes revenue structure of inventors, such as monopolistic rents. Nakamura [25] had proposed a mixed duopoly competition model with heterogeneous commodities. And either nationalized enterprise or private enterprise, both their timing of setting motivated coefficient and content of treaties are decided by owners of enterprise.

\section{Local Stability Analysis around Equilibrium Point}

3.1. Local Stability Analysis of the Nash Equilibrium Point. Before discussing local stability property of equilibrium points, the following symbols, which are $A_{1}=2 b+2 c-\alpha_{1} b$ and $A_{2}=2 b+2 c-\alpha_{2} b$, should be defined to facilitate the calculation. As the parameters $b$ and $c$ are both positive, and the parameter $\alpha_{i}$ meets $0 \leq \alpha_{i} \leq 1$, so we can deduce that

$$
A_{i}>0, A_{i}-b>0, \quad i=1,2 .
$$

Let $T\left(q_{1}, q_{2}\right)=\left(q_{1}, q_{2}\right)$ in system (11), then we can get three boundary equilibrium points $E_{0}=(0,0)$, $E_{1}=\left(0,\left(a / A_{2}\right)\right)$, and $E_{2}=\left(\left(a / A_{1}\right), 0\right)$, and a unique Nash equilibrium point $E^{*}=\left(\left(a\left(A_{1}-b\right) /\left(A_{1} A_{2}-b^{2}\right)\right)\right.$, $\left.\left(a\left(A_{2}-b\right) /\left(A_{1} A_{2}-b^{2}\right)\right)\right)$. According to the economic significance of all the parameters, the equilibrium points of system (11) are nonnegative as long as the following condition holds:

$$
A_{1} A_{2}-b^{2}>0 \text {. }
$$

Through calculating the eigenvalue of characteristic matrix, also called the Jacobi matrix, we could easily get stable condition about the equilibrium solution of mapping (11). The Jacobi matrix of mapping $(11)$ at $\left(q_{1}, q_{2}\right)$ is given as

$$
J\left(q_{1}, q_{2}\right)=\left(\begin{array}{cc}
1+v_{1} a-2 v_{1} A_{1} q_{1}-v_{1} b q_{2} & -v_{1} b q_{1} \\
-v_{2} b q_{2} & 1+v_{2} a-2 v_{2} A_{2} q_{2}-v_{2} b q_{1}
\end{array}\right) .
$$

First of all, we get the following theorems about the boundary equilibrium points $E_{0}, E_{1}$, and $E_{2}$.

Theorem 1. $E_{0}$ is an unstable node.

Proof. Through calculating the corresponding Jacobi matrix at $E_{0}$, we can get

$$
J\left(E_{0}\right)=\left(\begin{array}{cc}
1+v_{1} a & 0 \\
0 & 1+v_{2} a
\end{array}\right) .
$$

Obviously, the eigenvalues of the above matrix are the elements on the diagonal, i.e., $\lambda_{i}=1+v_{i} a,(i=1,2)$. As the parameters meet $v_{i}>0,(i=1,2)$ and $a>0$, it is easy to know that $\lambda_{i}>1$. Hence, $E_{0}$ is always an unstable node. 
Theorem 2. If the parameters meet $0<v_{2}<(2 / a), E_{1}$ is a saddle. While if parameter condition meets $v_{2}>(2 / a), E_{1}$ is an unstable node.

Proof. Similarly, through calculating the corresponding Jacobi matrix at $E_{1}$, we can know that

$$
J\left(E_{1}\right)=\left(\begin{array}{cc}
1+v_{1} a-\frac{v_{1} a b}{A_{2}} & 0 \\
-\frac{v_{2} a b}{A_{2}} & 1-v_{2} a
\end{array}\right) .
$$

It is obvious that the eigenvalues of the above matrix are $\lambda_{1}=1+\left(v_{1} a\left(A_{2}-b\right) / A_{2}\right)$ and $\lambda_{2}=1-v_{2} a$. Because the parameters meet $a>0, v_{i}>0(i=1,2)$, and $A_{i}-b>0$, we can easily know that $\lambda_{1}>1$ always holds. If the parameters satisfy $0<v_{2}<(2 / a)$, we can infer that $\left|\lambda_{2}\right|<1$, so $E_{1}$ is a saddle point. While if the parameters satisfy $v_{2}>(2 / a)$, we can deduce that $\left|\lambda_{2}\right|>1$. So, $E_{1}$ is an unstable node at such a situation.

Analogically, we can conclude that $E_{2}$ is a saddle, when $0<v_{1}<(2 / a)$. While if $v_{1}>(2 / a), E_{2}$ is an unstable node.
Regarding the local stability property of all boundary equilibrium points, we have discussed clearly. The local stability at the unique internal equilibrium solution $E^{*}=$ $\left(q_{1}^{*}, q_{2}^{*}\right)$ will be analyzed in the following discussion, where $q_{1}^{*}=\left(a\left(A_{1}-b\right) /\left(A_{1} A_{2}-b^{2}\right)\right)$ and $q_{2}^{*}=\left(a\left(A_{2}-b\right) /\left(A_{1}\right.\right.$ $\left.\left.A_{2}-b^{2}\right)\right)$. At $E^{*}=\left(q_{1}^{*}, q_{2}^{*}\right)$, the specific form of matrix (14) is given as

$$
J\left(E^{*}\right)=\left(\begin{array}{cc}
1+v_{1} q_{1}^{*}\left(A_{2}-2 A_{1}\right) & -v_{1} b q_{1}^{*} \\
-v_{2} b q_{2}^{*} & 1+v_{2} q_{2}^{*}\left(A_{1}-2 A_{2}\right)
\end{array}\right) .
$$

Quite evidently, the trace ( $\mathrm{Tr}$ ) and determinant (Det) of above matrix $J\left(E^{*}\right)$ can be expressed as

$$
\begin{aligned}
\operatorname{Tr}= & 2+v_{1} q_{1}^{*}\left(A_{2}-2 A_{1}\right)+v_{2} q_{2}^{*}\left(A_{1}-2 A_{2}\right), \\
\operatorname{Det}= & 1+v_{1} q_{1}^{*}\left(A_{2}-2 A_{1}\right)+v_{2} q_{2}^{*}\left(A_{1}-2 A_{2}\right) \\
& +v_{1} v_{2} q_{1}^{*} q_{2}^{*}\left(A_{1} A_{2}-b^{2}-2\left(A_{1}-A_{2}\right)^{2}\right) .
\end{aligned}
$$

According to the Jury criterion, the stability region of $E^{*}=\left(q_{1}^{*}, q_{2}^{*}\right)$ is bounded by the curves given by following inequalities (19), and these three inequalities are also the stable conditions of $E^{*}=\left(q_{1}^{*}, q_{2}^{*}\right)$ :

$$
\left\{\begin{array}{l}
A_{1} A_{2}-b^{2}-2\left(A_{1}-A_{2}\right)^{2}>0, \\
4+2 v_{1} q_{1}^{*}\left(A_{2}-2 A_{1}\right)+2 v_{2} q_{2}^{*}\left(A_{1}-2 A_{2}\right)+v_{1} v_{2} q_{1}^{*} q_{2}^{*}\left(A_{1} A_{2}-b^{2}-2\left(A_{1}-A_{2}\right)^{2}\right)>0 \\
v_{1} q_{1}^{*}\left(A_{2}-2 A_{1}\right)+v_{2} q_{2}^{*}\left(A_{1}-2 A_{2}\right)+v_{1} v_{2} q_{1}^{*} q_{2}^{*}\left(A_{1} A_{2}-b^{2}-2\left(A_{1}-A_{2}\right)^{2}\right)<0 .
\end{array}\right.
$$

3.2. Numerical Analysis. The local stability property around points $E_{0}, E_{1}$, and $E_{2}$ has been analyzed above, and then we can draw stability region of $E^{*}=\left(q_{1}^{*}, q_{2}^{*}\right)$ through the Jury criterion given in equation (19). Next, we could analyze different paths to chaos and the impact of different parameters on the stability region of $E^{*}=\left(q_{1}^{*}, q_{2}^{*}\right)$ using the numerical method. The parameters will be fixed as $a=5.431$, $b=0.004, c=2.344, \alpha_{1}=0.002$, and $\alpha_{2}=0.995$ in Figure 1 , and the corresponding 2-D bifurcation diagram about $v_{1}$ and $v_{2}$ is given in Figure 1(a). From Figure 1(a), we can see that stability region of $E^{*}=\left(q_{1}^{*}, q_{2}^{*}\right)$ just looks like a standard square (see the region of dark goldenrod in Figure 1(a) for more details). We can also see from Figure 1(a) that system (11) can only enter chaos through period-doubling bifurcation with the increase of adjusting speed. However, we cannot find the fractal structure from the 2-D bifurcation diagram, which means that the dynamical behavior of system (11) is relative simple at such a group of parameters. However, if we reset the system parameters as $a=6.5, b=1.2, c=0.5, \alpha_{1}=0.002$, and $\alpha_{2}=0.995$, the corresponding two-parameter bifurcation diagram is shown in Figure 1(b). Compared with Figure 1(a), it is not difficult to find from Figure 1(b) that not only the size of stability region has increased, but also the shape of the stability region has also changed. However, what has unchanged is that the system can only undergo perioddoubling bifurcation.

The 2-D bifurcation diagrams in Figure 1 are rather simple, but if we further change the system parameters, the dynamics of mapping (11) may become quite complicated. For instance, when we choose parameters as $a=6.977$, $b=0.325, c=1.815, \alpha_{1}=0.677$, and $\alpha_{2}=0.583$, we can find $E^{*}=\left(q_{1}^{*}, q_{2}^{*}\right)$ will lose its stability by a flip bifurcation, and then a pair of stable fixed points and stable period-4 fixed point will come to arise one after another after this kind bifurcation. However, unlike Figure 1, the stable period-4 state of system (11) can also lose stability through a Neimark-Sacker bifurcation, which can be seen from the narrow gap between the yellow areas in Figure 2(a). In order to further reveal the complex dynamical behavior of system (11) under such a set of parameters, Figure 2(a) is partially enlarged, which can be seen from Figure 2(b). We can see a very beautiful fractal structure from Figure 2(b), and it looks like a shield. In addition, we can also see a jellyfish surrounded by many Arnold tongues. If we further change the parameters to $a=7.212, b=0.28, c=1.1, \alpha_{1}=0.699$, and $\alpha_{2}=0.191$, we can find that the shape of stability region of the Nash equilibrium point has changed, and the window of Neimark-Sacker bifurcation has also increased. In order to reveal the fractal structure of Figure 2(c) more clearly, it has 


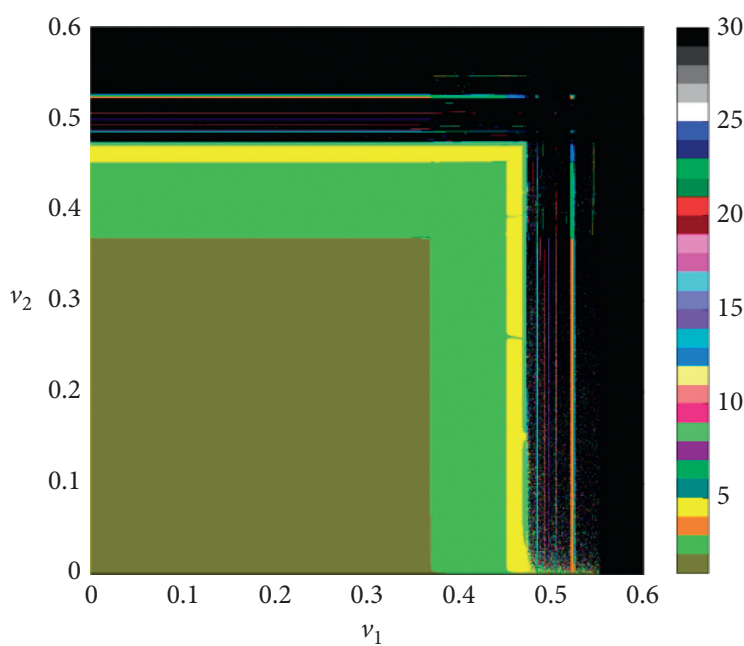

(a)

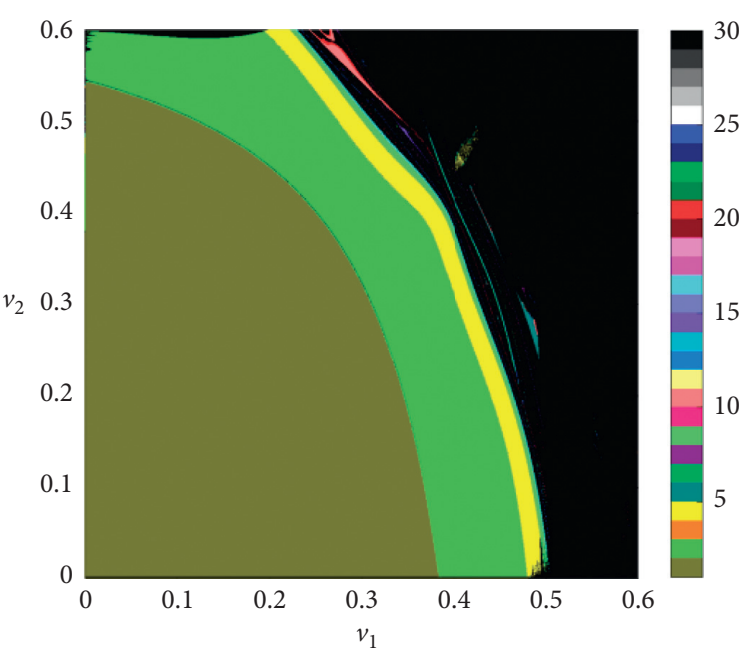

(b)

FIGURE 1: The 2-D bifurcation diagrams about $v_{1}$ and $v_{2}$, where the rest parameters are fixed as follows: (a) $a=5.431, b=0.004, c=2.344$, $\alpha_{1}=0.002$, and $\alpha_{2}=0.995$; (b) $a=6.5, b=1.2, c=0.5, \alpha_{1}=0.002$, and $\alpha_{2}=0.995$.

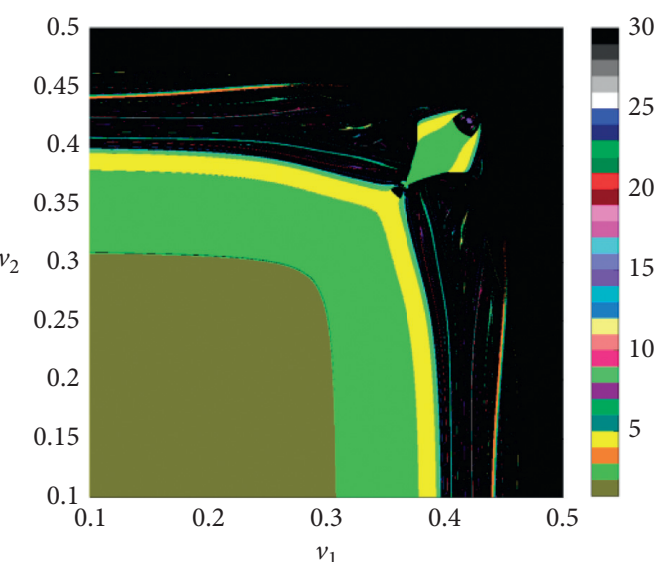

(a)

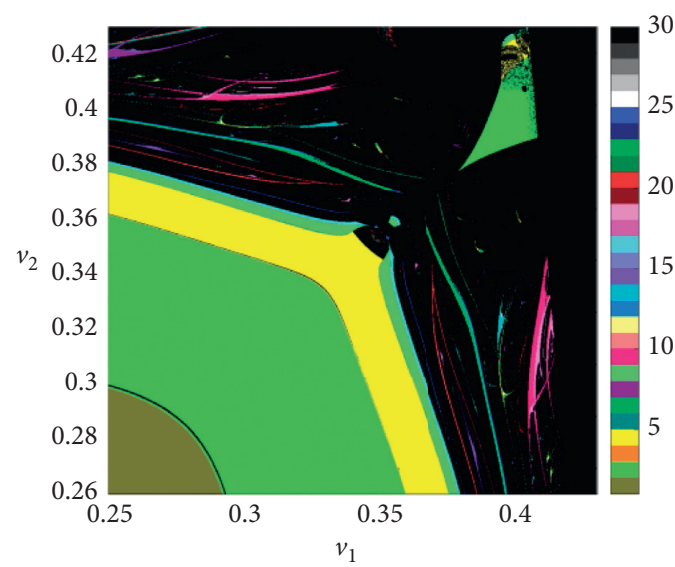

(c)

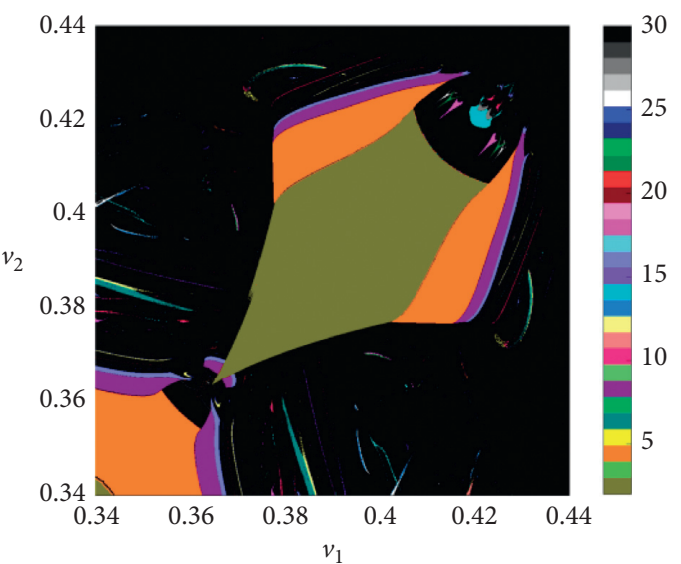

(b)

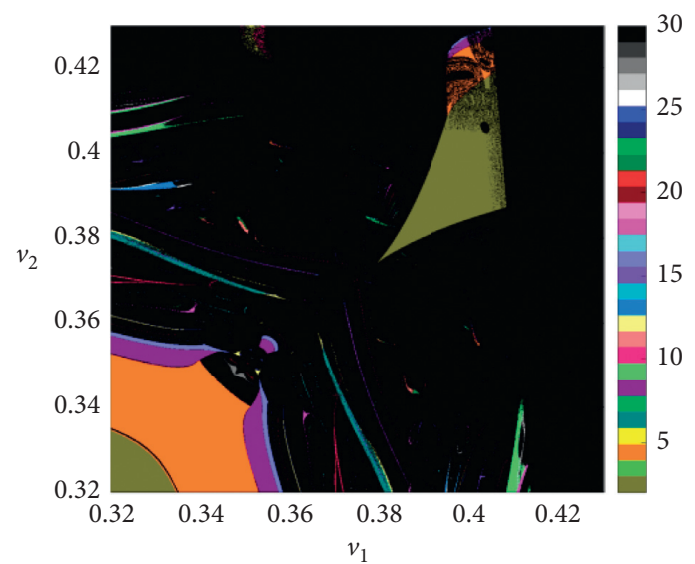

(d)

Figure 2: The 2-D bifurcation diagrams about $v_{1}$ and $v_{2}$, where the rest parameters are fixed as follows: (a) $a=6.977, b=0.325, c=1.815$, $\alpha_{1}=0.677$, and $\alpha_{2}=0.583$; (b) partial enlargement of Figure 2(a); (c) $a=7.212, b=0.28, c=1.1, \alpha_{1}=0.699$, and $\alpha_{2}=0.191$; (d) partial enlargement of Figure 2(c). 
also been partially enlarged, see Figure 2(d) for more details. From Figure 2(d), we can see that the outline of the shield still exists, but its main part has been covered with black color. It is just like a beautiful girl wearing a black veil. And behind the veil is the coexistence of multiple attractors, which will be discussed in the following section.

Previously, we have discussed the impact of enterprises' adjusting speed on the system dynamical behaviors, and then we will continue to discuss the impact of enterprises' public ownership (namely, $\alpha_{1}$ and $\alpha_{2}$ ) on the system dynamics. Figure 3 shows the 2-D bifurcation diagram of system (11) about the degree of public ownership of these two enterprises. Obviously, Figure 3 is not as rich as Figures 1 and 2 in dynamics. That is to say, compared with the speed of adjustment, the impact of the degree of public ownership on the system's dynamical behavior is not very prominent. From Figure 3, we can only see many regular periodic bands. In particular, we can find that the increase of enterprise 1's public ownership degree or the decrease of enterprise 2's public ownership degree can make the system lose stability, when the adjusting speed of enterprise 1 is smaller than that of enterprise 2, see Figure 3(a) for details. However, the increase of enterprise 1's public ownership degree or the decrease of enterprise 2's public owner ship degree will make the system more stable, when the adjusting speed of enterprise 1 is larger than that of enterprise 2 . Therefore, we can conclude that the larger the adjusting speed of enterprise with higher degree of public ownership is, the more stable the system is.

In summary, based on several sets of different parameters, the dynamical behaviors of the system in the twodimensional parameter space $\left(v_{1}, v_{2}\right)$ and $\left(\alpha_{1}, \alpha_{2}\right)$ are discussed. And the following actual significance of the given system can be obtained:

(1) When the elasticity coefficient of market supply and demand and the coefficient of public ownership degree of enterprises are taken different, the twoparameter bifurcation diagrams in $\left(v_{1}, v_{2}\right)$ will also be different. The stability is increased with the reduction of the speed of enterprise regulation.

(2) With the increase of public ownership, the system will be more stable. And the enterprises with higher degree of public ownership tend to pay more attention to the implementation of intellectual property policies.

There is widespread concern about the relationship between innovation and corporate performance. Enterprises attempt to create sustainable competitive advantages by effectively utilizing their resources and capabilities.
Managers who ignore to protect IPR essentially discourage innovation effort [26].

In general, private and small businesses have fewer patents due to the lack of appropriate intellectual property rights and the cost of obtaining and managing patents [27]. Innovation is a key driver of business performance because it allows manufacturing companies to transform their dynamic capabilities to learn and leverage new ideas, skills, and capabilities. Previous innovation studies have often focused on the link between innovation and company performance [28]. As a result, there is still little clarity about the exact mechanism by which IPR may affect the relationship between innovation potential and enterprise performance in a highly competitive market place. Furukawa [29] reported novel empirical evidence that enhancing intellectual property rights (IPRs) protection reduces innovation activities when IPR protection is already strong. This suggests that the relationship between IPR protection and innovation is shaped like an inverted-U.

\section{Global Dynamics Analysis}

4.1. Noninvertible Map and Critical Curves. The properties of the inverse of a map play an important role in understanding the changes in the structure of the attracting domain. The model established in this research is a difference equation, also known as a map of the dynamical system. Generally speaking, the map of dynamical system can be divided into invertible map and noninvertible map. If different points in the state space have the same image, that is, there exists $Q_{1} \neq Q_{2}$ making $T\left(Q_{1}, \bar{\lambda}\right)=T\left(Q_{2}, \bar{\lambda}\right)=Q^{\prime}$, then the map is called a noninvertible map. Here, $Q^{\prime}$ is the image of both $Q_{1}$ and $Q_{2}$, and $Q_{1}$ and $Q_{2}$ are two different preimages of $Q^{\prime}$. Obviously, the phase space of noninvertible map can also be divided into different sets according to the number of preimages. In general, we use the symbol $Z_{i},(i \in N)$ to represent the set of points with $i$ preimages in the phase space, and the dividing lines between those different sets are called critical curves, which are labeled by LC. The rank-1 preimage of $\mathrm{LC}$ under the map $T$ is recorded as $\mathrm{LC}_{-1}$. Actually, the critical curve LC is the extension of the local maximum of the one-dimensional noninvertible map in the multidimensional map, and its preimage $\mathrm{LC}_{-1}$ corresponds to the local extreme point of the one-dimensional map. Especially, when the noninvertible map $T$ is continuous and differentiable, $\mathrm{LC}_{-1}$ is usually contained in the point set that makes the Jacobi determinant of $T$ to zero. Obviously, system (11) is a continuous differentiable map, and the points where the determinant of matrix (14) is zero must satisfy the following equation:

$$
\begin{gathered}
2 v_{1} v_{2} b A_{1} q_{1}^{2}+2 v_{1} v_{2} b A_{2} q_{2}^{2}+4 v_{1} v_{2} b A_{1} A_{2} q_{1} q_{2}-\left(v_{2} b+v_{1} v_{2} a b+2 v_{1} A_{1}+2 v_{1} v_{2} a A_{1}\right) q_{1} \\
-\left(v_{1} b+v_{1} v_{2} a b+2 v_{2} A_{2}+2 v_{1} v_{2} a A_{2}\right) q_{2}+v_{1} v_{2} a^{2}+v_{1} a+v_{2} a+1=0
\end{gathered}
$$

where $A_{1}=2 b+2 c-\alpha_{1} b$ and $A_{2}=2 b+2 c-\alpha_{2} b$. By solving the above equation, it can be seen that the critical curve $\mathrm{LC}_{-1}$ is composed of two disconnected parts, namely, $\mathrm{LC}_{-1}=\mathrm{LC}_{-1}^{(a)} \cup \mathrm{LC}_{-1}^{(b)}$. Therefore, the critical curve 


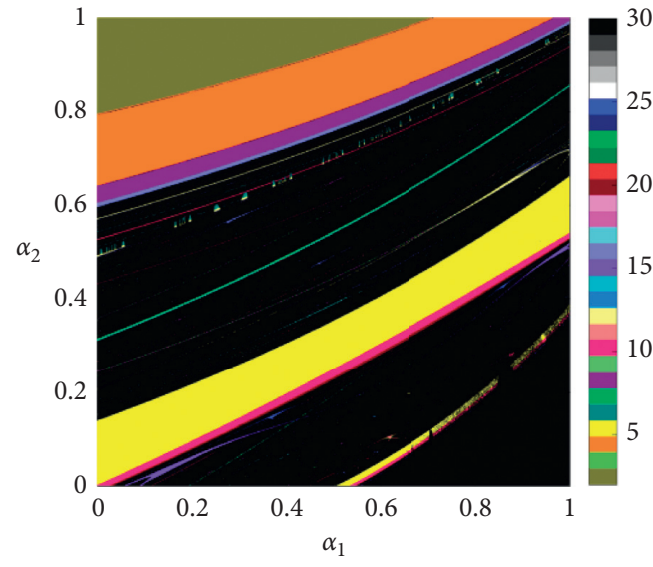

(a)

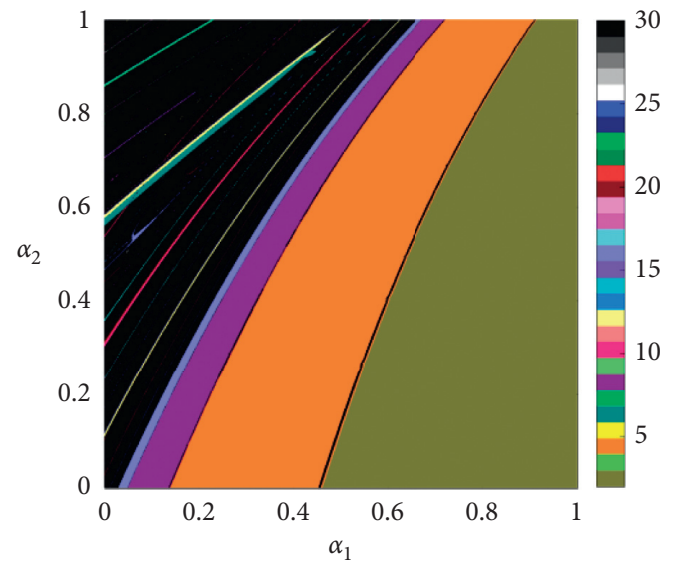

(b)

FiguRE 3: The 2-D bifurcation diagrams about $\alpha_{1}$ and $\alpha_{2}$, where the rest parameters are fixed as follows: (a) $a=4.69976212, c=0.36680549$, $b=0.92281351, v_{1}=0.26457329$, and $v_{2}=0.777$; (b) $a=4.8208, c=0.0425, b=0.1322, v_{1}=0.6524$, and $v_{2}=0.3301$.

$\mathrm{LC}=T\left(\mathrm{LC}_{-1}\right)$ also has two branches, i.e., $\mathrm{LC}^{(a)}=T\left(\mathrm{LC}_{-1}^{(a)}\right)$ and $\mathrm{LC}^{(b)}=T\left(\mathrm{LC}_{-1}^{(b)}\right)$ (see Figure 4 for more details). The two branches of the critical curve have different meanings. For example, $\mathrm{LC}^{(b)}$ separates the region without the rank-1 preimage $Z_{0}$ from the region with two rank-1 preimages $Z_{2}$, while $\mathrm{LC}^{(a)}$ separates the region with two rank-1 preimages $Z_{2}$ from the region with four rank-1 preimages $Z_{4}$. Actually, as the origin $O$ lies in the region $Z_{4}$, it has four preimages. If we let $q_{1}^{\prime}=0$ and $q_{2}^{\prime}=0$ in system (11), then the following equations can be obtained:

$$
\left\{\begin{array}{l}
q_{1}+v_{1} q_{1}\left[a-b q_{2}-\left(2 b+2 c-\alpha_{1} b\right) q_{1}\right]=0 \\
q_{2}+v_{2} q_{2}\left[a-b q_{1}-\left(2 b+2 c-\alpha_{2} b\right) q_{2}\right]=0
\end{array}\right.
$$

By solving equations (21), we can get four preimages of the origin $O$, which are $O, O_{-1}^{1}=\left(\left(\left(1+v_{1} a\right) /\right.\right.$ $\left.\left.v_{1}\left(2 b+2 c-\alpha_{1} b\right)\right), 0\right), \quad O_{-1}^{2}=\left(0,\left(\left(1+v_{2} a\right) / \quad v_{2}(2 b+2 c-\right.\right.$ $\left.\left.\alpha_{2} b\right)\right)$ ) and the intersection of $\omega_{1}^{-1}$ and $\omega_{2}^{-1}$, namely, $O_{-1}^{3}$. And $\omega_{1}^{-1}$ and $\omega_{2}^{-1}$ are the rank-1 preimages of $\omega_{1}$ and $\omega_{2}$, which are two line segments on the $x$-axis and the $y$-axis, respectively.

4.2. Invariant Set. It is easy to prove that $T\left(q_{1}, 0\right)=\left(q_{1}^{\prime}, 0\right)$ and $T\left(0, q_{2}\right)=\left(0, q_{2}^{\prime}\right)$, so the two axes of phase space are invariant sets. And hence, the dynamical behaviors of system (11) on the axes can be discussed by the following one-dimensional map $q_{i}^{\prime}=f_{i}\left(q_{i}\right),(i=1,2)$, and the specific form of map $f_{i},(i=1,2)$ can be expressed as

$$
q_{i}^{\prime}=q_{i}+v_{i} q_{i}\left(a-\left(2 b+2 c-\alpha_{i} b\right) q_{i}\right), \quad i=1,2 .
$$

Through the following linear transformation,

$$
q_{i}=\frac{1+v_{i} a}{v_{i}\left(2 b+2 c-\alpha_{i} b\right)} \omega, \quad i=1,2 .
$$

We can find that the map $f_{i},(i=1,2)$ is equivalent to the logistic map $u=\lambda_{i} u(1-u),(i=1,2)$, where

$$
\lambda_{i}=1+v_{i} a, \quad i=1,2 .
$$

Therefore, the trajectory properties of system (11) on the invariant axis can be easily obtained by studying the logistic map.

Theorem 3. There exists a threshold of enterprise is adjusting speed $v_{i},(i=1,2)$, namely, $\bar{v}_{i}=(3 / a)$ (or a threshold $\bar{a}$ of $a)$. And for any $v_{i} \in((3 / a),+\infty),(i=1,2)$ (or for any $\left.a \in\left(\left(3 / v_{i}\right),+\infty\right)\right)$, the trajectories of nonlinear system (11) on the invariant axes will diverge.

Proof. It has been shown that the dynamical behaviors of system (11) on the invariant axes are controlled by onedimensional map (22), which is also topologically equivalent to the standard logistic map. According to the properties of the logistic map, we know that the trajectories will diverge, when $\lambda_{i}>4,(i=1,2)$. Through equation (24), we can easily know that this condition can be transformed into $v_{i} a>3,(i=1,2)$. So, the theorem is proved.

4.3. GlobalDynamics Analysis. If we record the feasible set of system (11) as $B$, then the feasible trajectory of system (11) in feasible set $B$ may eventually converge to the Nash equilibrium point $E^{*}$, or it will converge to other more complex attractors. However, the trajectory starting from the outside of feasible set shows the "blowout" evolution of the economic system. In general, the feasible set of a nonlinear system often has fractal boundary, and the fractal boundary will also change with the change of parameters. In order to analyze the forming process of fractal boundary of the feasible region, we first discuss the dynamical behaviors of map $T$ on the invariant axes. According to Theorem 3, we know that the trajectories from the line segments $\omega_{i}=\left(O, O_{-1}^{i}\right)$ and $(i=1,2)$ will be bounded, where $O_{-1}^{i}$ and $(i=1,2)$ are the rank-1 preimages of the origin. And the feasible region of the system is just the bounded region formed by the line segments $\omega_{i}=\left(O, O_{-1}^{i}\right)$ and their rank-1 preimages $\omega_{i}^{-1}=T^{-1}\left(\omega_{i}\right)$. Actually, if we choose any point $(p, 0),\left(0<p<\left(\left(1+v_{1} a\right) / v_{1}\left(2 b+2 c-\alpha_{1} b\right)\right)\right)$ from the line 


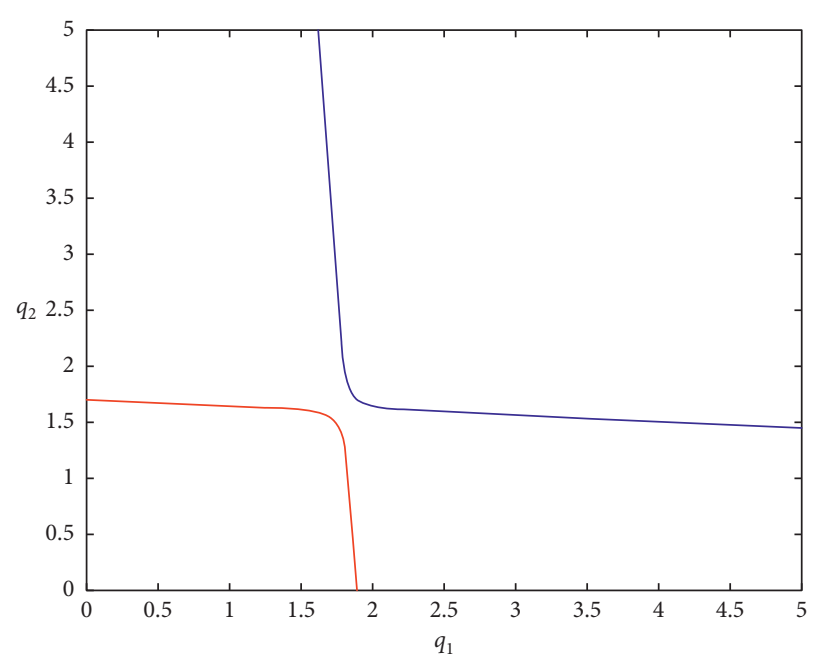

(a)

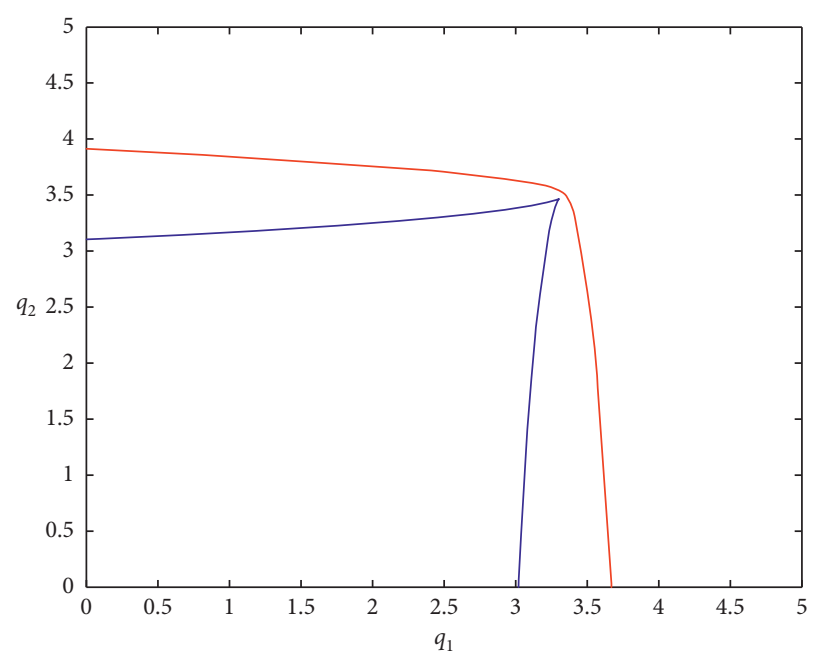

(b)

FIgURE 4: Schematic diagram of the critical curves, where the parameters are chosen as $a=7.212, b=0.28, c=1.1, \alpha_{1}=0.699, \alpha_{2}=0.191$, $v_{1}=0.4$, and $v_{2}=0.5$.

segment $\omega_{1}$, then the rank-1 preimages of $(p, 0)$ are determined by the following algebraic equations:

$$
\left\{\begin{array}{l}
q_{1}+v_{1} q_{1}\left[a-b q_{2}-\left(2 b+2 c-\alpha_{1} b\right) q_{1}\right]=p, \\
q_{2}+v_{2} q_{2}\left[a-b q_{1}-\left(2 b+2 c-\alpha_{2} b\right) q_{2}\right]=0 .
\end{array}\right.
$$

It is clear that the point $(p, 0)$ has two distinct rank-1 preimages, one of which is located on the line segment $\omega_{1}$, and the other is determined by the following equation:

$$
q_{1}=\left(\alpha_{2}-2-\frac{2 c}{b}\right) q_{2}+\frac{1+v_{2} a}{b v_{2}} .
$$

Obviously, the straight line determined by (26) is just the rank-1 preimage of $\omega_{1}$, which is labeled by $\omega_{1}^{-1}$. Similarly, the rank-1 preimage of any point $(0, q), \quad(0<q<((1+$ $\left.\left.\left.v_{2} a\right) / v_{2}\left(2 b+2 c-\alpha_{2} b\right)\right)\right)$ on the line segment $\omega_{2}$ is either located on the invariant axis or determined by the following equation:

$$
q_{2}=\left(\alpha_{1}-2-\frac{2 c}{b}\right) q_{1}+\frac{1+v_{1} a}{b v_{1}} .
$$

It is clear that the straight line determined by (27) is the rank-1 preimage of $\omega_{2}$, which is labeled by $\omega_{2}^{-1}$. It is worth noting that straight line (26) intersects the $q_{2}$-axis at the point $\mathrm{O}_{-1}^{2}$ and straight line (27) intersects the $q_{1}$-axis at the point $\mathrm{O}_{-1}^{1}$, and the intersection of straight line (26) and the straight line (27) is $\mathrm{O}_{-1}^{3}$. According to the above analysis, we can draw the conclusion as the boundary of feasible set is the union of invariant axes and their preimages of any rank. It means that

$$
\partial B(\infty)=\left(\bigcup_{k=0}^{\infty} T^{\prime(-k)}\left(\omega_{1}\right)\right) \bigcup\left(\bigcup_{k=0}^{\infty} T^{\prime(-k)}\left(\omega_{2}\right)\right) .
$$

The invariant sets and critical curves of a noninvertible map can be used to analyze the change of attracting basins with varying parameters. In order to explain the global dynamical behaviors of system (11) with different values of $v_{1}$ and $v_{2}$, we fix the system parameters as $a=4.69976212$, $b=0.92281351, \alpha_{1}=0.066, \alpha_{2}=0.621$, and $c=0.36680549$. When we choose the values of parameters $v_{1}$ and $v_{2}$ as 0.26457329 and 0.49 , respectively, the relative relation among the invariant sets, the critical curves, and feasible regions is shown in Figure 5(a). From Figure 5(a), we can see that the rank-1 preimage of $\omega_{i}$, i.e., $\omega_{i}^{-1}$ belongs to the region of $Z_{0}$, it implies that $\omega_{1}$ and $\omega_{2}$ have only the rank-1 preimages, and thus, the attracting basin of the Nash equilibrium point is just a simple connected set, and the boundary of the attracting basin is also very simple. According to Theorem 3, we know that the critical curve begins to contact with the boundary of the attracting basin when $v_{i}=(3 / a)$, $(i=1,2)$. Actually, if we fix the parameters as $v_{2}=(3 / a)$, we can find that one of the branches of the critical curves, i.e., $\mathrm{LC}^{(b)}$ begins to contact with the boundary $\omega_{1}^{-1}$, the Nash equilibrium point will become an unstable saddle, and a period-2 cycle will come arise. As we can see from Figure 5 (b) that part of $B(\infty)$ has entered $Z_{2}$ from $Z_{0}$, which is labeled by $h_{0}$ and is also called main "hole," and two rank-1 preimages of $h_{0}$, i.e., $h_{-1}^{1}$ and $h_{-1}^{2}$ begin to emerge in the feasible region. As the value of $v_{2}$ further increases, we can find that the main "hole" will also increase further. As main "hole" $h_{0}$ belongs to $Z_{2}$, we can find two rank- 1 preimages of $h_{0}$, i.e., $h_{-1}^{1}$ and $h_{-1}^{2}$. As $h_{-1}^{1}$ and $h_{-1}^{2}$ belong to belong to $Z_{2}$ and $Z_{4}$, respectively, we can see that they have two or four different rank-1 preimages. And these preimages also have many preimages of high rank, so we can find a series of holes in the boundary of the feasible region, and the simple connected basin is also transformed into a complex connected basin (see Figure 5(c)). With the further increase of $v_{2}$, the critical curve LC continues to move upward, and the main "hole" becomes larger and larger. It means that the probability of choosing the initial conditions from the holes becomes larger, and the economic system also loses 


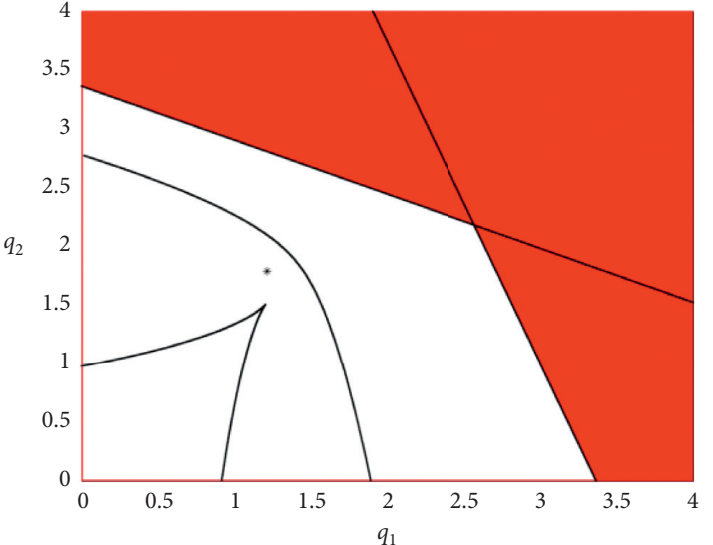

(a)

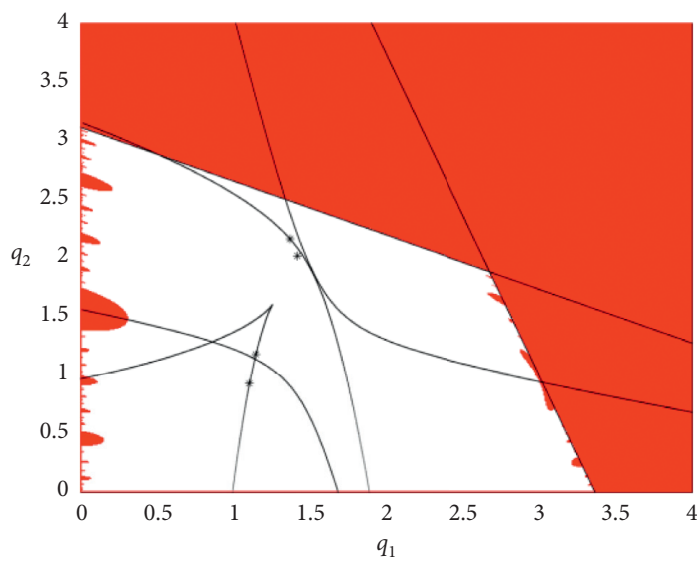

(c)

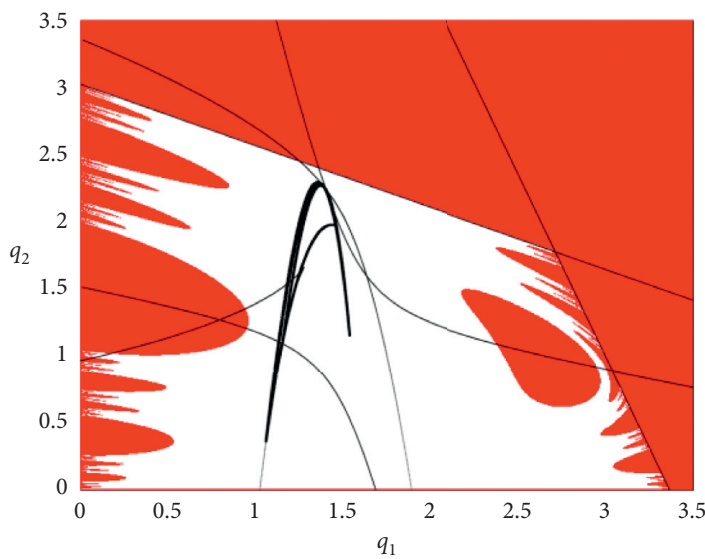

(e)

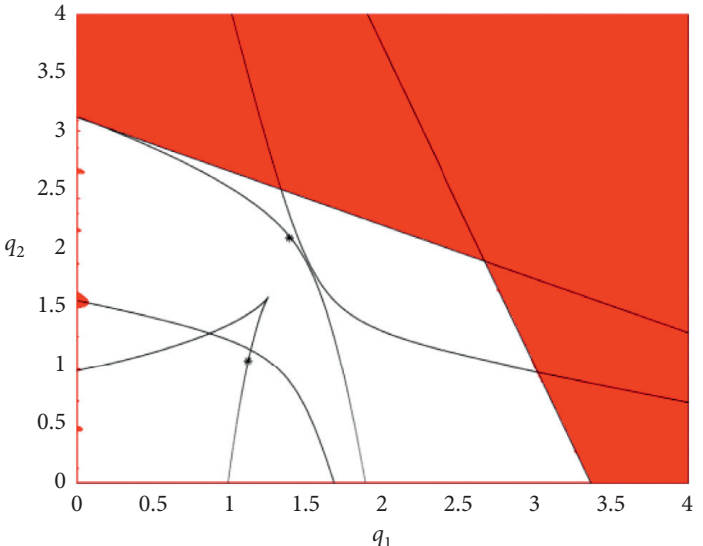

(b)

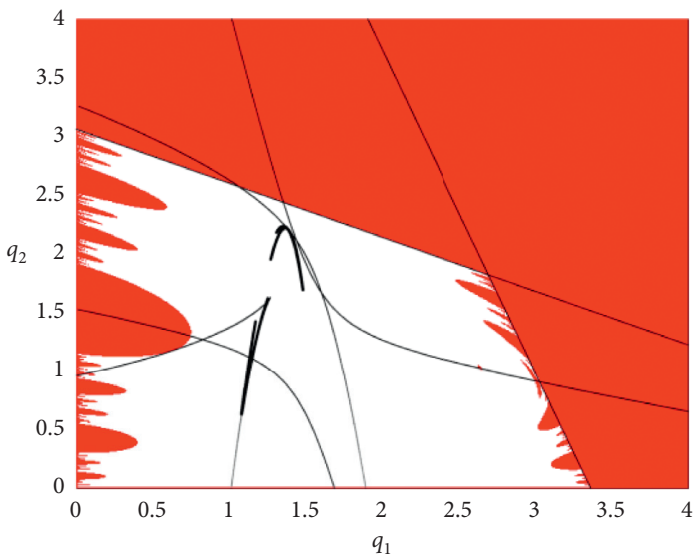

(d)

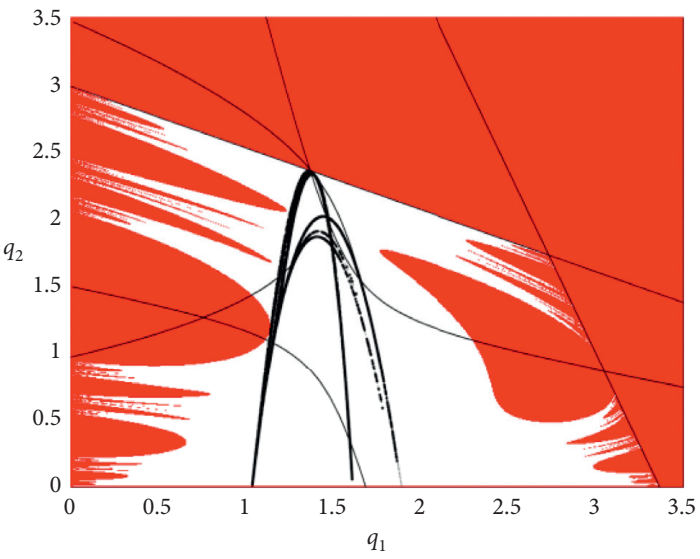

(f)

FIGURE 5: The evolution of the attractor and its attracting basin, where the parameters are fixed as $a=4.69976212, b=0.92281351$, $\alpha_{1}=0.066, \alpha_{2}=0.621, c=0.36680549$, and $v_{1}=0.26457329$ : (a) $v_{2}=0.49 ;$ (b) $v_{2}=0.64 ;$ (c) $v_{2}=0.65$; (d) $v_{2}=0.695$; (e) $v_{2}=0.733$; (f) $v_{2}=0.777$.

predictability. At the same time, two pieces of the chaotic attractor (see Figure 5(d)) or a whole chaotic attractor (see Figure 5(e) and 5(f)) will be generated through a flip bifurcation sequence. Especially, in Figure 5(f), we can see that the chaotic attractor has contacted the boundary of its basin, it implies that a global bifurcation called "contact" bifurcation will happen, and the chaotic attractor will be destroyed. In fact, there is no connection between these two contact bifurcations mentioned above. That is, the attracting basin of a simple attractor (such as a fixed point or a periodic cycle) may have complex structure, or the structure of a chaotic attractor may be very simple. As discussed in many literature studies, the increase of adjustment speed will lead to system instability and unpredictability. However, we also 
find that the increase of adjusting speed will lead to contact bifurcation.

\section{Conclusion}

In this research, we reconstructed a new mixed duopoly game model based on the oligopoly game theory and the intellectual property rights protection policy. And the complex dynamical behaviors of the given model were also investigated by the two-dimensional bifurcation diagrams. The bifurcation diagrams in the parameter space $\left(v_{1}, v_{2}\right)$ and $\left(\alpha_{1}, \alpha_{2}\right)$ reveal that the dynamic behavior of the system can be very complex. These dynamical properties can present the long-run effects of strengthening intellectual property protection.

The essence of intellectual property protection is to grant the innovators monopoly interests in a certain period of time so as to encourage them to invest knowledge, time, and capital in innovation activities. Under the condition of constant innovation cost, the net income of innovation increases, and the enthusiasm of enterprise innovation also increases. On the other hand, intellectual property protection increases the cost of imitation by other enterprises.

In a word, no matter from the perspective of improving the expected income of innovative enterprises, or from the perspective of increasing the imitation cost of backward enterprises to reduce imitation, strengthening intellectual property rights protection will provide innovation power for enterprises with strong innovation ability.

However, the increase in the intensity of intellectual property protection will increase the cost of technology transfer and reduce the income transferee enterprises as well as social welfare. Therefore, while increasing the intensity of intellectual property protection, it is necessary to measure the monopoly effect and innovation effect of the innovative enterprises to make sure the innovation effect is greater than the monopoly effect, and to avoid the negative impact of intellectual property protection on social welfare in developing countries.

\section{Data Availability}

The authors state that no data were used in this research.

\section{Conflicts of Interest}

The authors declare that there are no conflicts of interest.

\section{Acknowledgments}

This project was supported by the Foundation of Humanities and Social Sciences from the Ministry of Education of China (project no. 15YJC820007), the Science and Technology Program of Gansu Province (no. 18ZC1LA008), and the Talents' Innovation and Entrepreneurship Project of Lanzhou City (no. 2015-RC-3).

\section{References}

[1] W. Yu and Y. Yu, "The complexion of dynamic duopoly game with horizontal differentiated products," Economic Modelling, vol. 41, pp. 289-297, 2014.
[2] W. Yu and Y. Yu, "The stability of Bayesian Nash equilibrium of dynamic Cournot duopoly model with asymmetric information," Communications in Nonlinear Science and Numerical Simulation, vol. 63, pp. 101-116, 2018.

[3] Y. Zhang, W. Zhou, T. Chu et al., "Complex dynamics analysis for a two-stage Cournot duopoly game of semi-collusion in production,” Nonlinear Dynamics, vol. 91, pp. 1-17, 2018.

[4] Y. Cao, W. Zhou, T. Chu, and Y. Chang, "Global dynamics and synchronization in a duopoly game with bounded rationality and consumer surplus," International Journal of Bifurcation and Chaos, vol. 29, no. 11, Article ID 1930031, 2019.

[5] B. Xin, F. Cao, W. Peng, and A. A. Elsadany, "A Bertrand Duopoly game with long- memory effects," Complexity, vol. 2020, Article ID 2924169, 7 pages, 2020.

[6] J. Zhou, W. Zhou, T. Chu, Y.-x. Chang, and M.-j. Huang, "Bifurcation, intermittent chaos and multi-stability in a twostage Cournot game with R\&D spillover and product differentiation," Applied Mathematics and Computation, vol. 341, pp. 358-378, 2019.

[7] T. Chen, B. Xiao, and H. Liu, "Credit risk contagion in an evolving network model integrating spillover effects and behavioral interventions," Complexity, vol. 2018, Article ID 1843792, 16 pages, 2018.

[8] T. Chen, Y. Wang, Q. Zeng, and J. Luo, "Network model of credit risk contagion in the interbank market by considering bank runs and the fire sale of external assets," Physica A: Statistical Mechanics and Its Applications, vol. 542, Article ID 123006, 2020.

[9] B. Xin and M. Sun, "A differential oligopoly game for optimal production planning and water savings," European Journal of Operational Research, vol. 269, no. 1, pp. 206-217, 2018.

[10] L. Xie and J. Ma, "Study the complexity and control of the recycling-supply chain of China's color TVs market based on the government subsidy," Communications in Nonlinear Science and Numerical Simulation, vol. 38, pp. 102-116, 2016.

[11] L. Xie, J. Ma, H. Han et al., "Implications of stochastic demand and manufacturers' operational mode on retailer's mixed bundling strategy and its complexity analysis," Applied Mathematical Modelling, vol. 55, pp. 484-501, 2018.

[12] E. L.-C. Lai and L. D. Qiu, "The north's intellectual property rights standard for the south?" Journal of International Economics, vol. 59, no. 1, pp. 183-209, 2003.

[13] G. M. Grossman and E. L.-C. Lai, "International protection of intellectual property: corrigendum," American Economic Review, vol. 96, no. 1, p. 456, 2006.

[14] G. M. Grossman and E. L.-C. Lai, "International protection of intellectual property," American Economic Review, vol. 94, no. 5, pp. 1635-1653, 2004.

[15] P.-C. Liao and K.-y. Wong, "R\&D subsidy, intellectual property rights protection, and north-south trade: how good is the TRIPS agreement?" Japan and the World Economy, vol. 21, no. 2, pp. 191-201, 2009.

[16] S. Wagner and S. Wakeman, "What do patent-based measures tell us about product commercialization? Evidence from the pharmaceutical industry," Research Policy, vol. 45, no. 5, pp. 1091-1102, 2016.

[17] I. Cockburn, J. Lanjouw, and M. Schankerman, "Patents and the global diffusion of new drugs," American Economic Review, vol. 106, no. 1, pp. 136-164, 2016.

[18] E. Billette de Villemeur, R. Ruble, and B. Versaevel, "Dynamic competition and intellectual property rights in a model of product development," Journal of Economic Dynamics and Control, vol. 100, pp. 270-296, 2019. 
[19] T. Chu, Study on Protection of Pharmaceutical Test Dada under the TRIPS Agreement, Intellectual Property Rights Publishing House Co., Ltd., Beijing, China, 2015.

[20] J. C. Bárcena-Ruiz and M. B. Garzón, "Privatisation and vertical integration under a mixed duopoly," Economic Systems, vol. 42, no. 3, pp. 514-522, 2018.

[21] C. Gutiérrez-Hita and J. Vicente-Pérez, "On supply function equilibria in a mixed duopoly," Economics Letters, vol. 168, pp. 6-9, 2018.

[22] Z. Yang, Y. Shi, and Y. Li, "Analysis of intellectual property cooperation behavior and its simulation under two types of scenarios using evolutionary game theory," Computers \& Industrial Engineering, vol. 125, pp. 739-750, 2018.

[23] G. I. Bischi and A. Naimzada, "Global analysis of a dynamic duopoly game with bounded rationality," Advances in $D y$ namics Game and Application, vol. 5, pp. 361-385, Birkhäuser, Boston, MA, USA, 2000.

[24] A. Barbu and G. Militaru, "The moderating effect of intellectual property rights on relationship between innovation and company performance in manufacturing sector," Procedia Manufacturing, vol. 32, pp. 1077-1084, 2019.

[25] Y. Nakamura, "Combining the endogenous choice of the timing of setting incentive parameters and the contents of strategic contracts in a managerial mixed duopoly," International Review of Economics \& Finance, vol. 59, pp. 207-233, 2019.

[26] M. A. Peteraf, "The cornerstones of competitive advantage: a resource-based view," Strategic Management Journal, vol. 14, no. 3, pp. 179-191, 1993.

[27] D. Cetindemas and G. Ulusoy, "Innovation performance and partnership in manufacturing firms in Turkey," Journal of Manufacturing Technology Management, vol. 19, no. 3, pp. 332-345, 2007.

[28] L. Raymond and J. St-Pierre, "R\&D as a determinant of innovation in manufacturing SMEs: an attempt at empirical clarification," Technovation, vol. 30, no. 1, pp. 48-56, 2010.

[29] Y. Furukawa, "Intellectual property protection and innovation: an inverted-U relationship," Economics Letters, vol. 109, no. 2, pp. 99-101, 2010. 